\title{
X-Ray CMOS Detector Array With Scintillating Light Guides
}

\author{
J. G. Rocha*, A. V. Fernandes, R. A. Dias, L. Goncalves, \\ G. Minas, P. Goncalves \\ Department of Industrial Electronics \\ University of Minho \\ Guimarães, Portugal \\ *gerardo@dei.uminho.pt
}

\begin{abstract}
This paper presents a preliminary study concerning an $x$-ray detector design to analyze a small area with high spatial resolution. The indirect method of $x$-ray detection is used. In this design, an array of scintillating CsI:Tl crystals, encapsulated in aluminum, is coupled with an array of photodetectors. This structure, patented and described by the authors in detail in previous works, can be obtained using the SU-8 negative photoresist as a sacrificial layer. The experimental work consisted in the deposition of a scintillator layer, and an aluminum layer, on the active area of a commercially available digital imaging sensor, thus supporting the developed detector design. X-ray imaging tests were performed using the PHILIPS $X$ 'Pert equipment. Promising results were obtained, featuring high resolution and detail.
\end{abstract}

\section{INTRODUCTION}

Digital radiography is widely replacing traditional radiography. As a major health care area, radiology is also an important research field. The x-ray detectors are currently undergoing fast development, towards the attainment of digital radiographies with improved spatial resolution while reducing the radiation dose applied to the patients. There are two main methods to construct x-ray detection systems, known as direct and indirect approaches [1]. The direct method normally uses a photoconductor that is directly exposed to the x-rays. In the indirect method, a scintillator is placed on the top of a photodetector. The scintillator absorbs the $\mathrm{x}$-ray energy and produces visible light, which is detected by the photodetector. A photodetector array is often used. This is a simple method but the spatial resolution encounters limitations due to scintillator thickness constraints (Fig. 1) [2].

Scintillating light guides separated by reflective surfaces can be used to increase the thickness of the scintillator without decreasing the spatial resolution (Fig. 2) [3]: the light yield by each scintillator is guided to the corresponding photodetector by the reflective walls.

\author{
A. J. Ferreira, S. Lanceros-Mendez \\ Department of Physics \\ University of Minho \\ Braga, Portugal
}

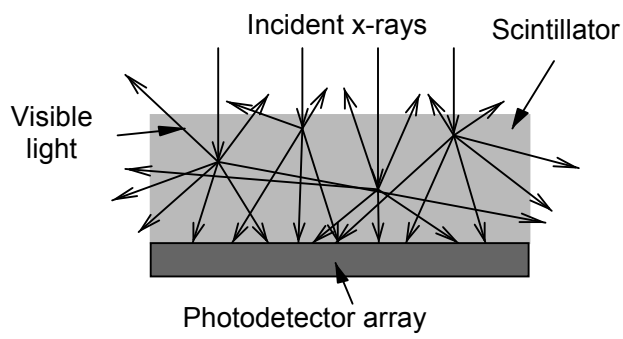

Figure 1. X-ray detector representation with a scintillator layer placed on top of a photodetector array.

There have been described some methods to produce light guides $[3,4,5]$. In this work we show a new approach based on the implementation of reflective aluminum walls.

\section{DEVICE DESIGN}

The fabrication steps of this $\mathrm{x}$-ray detector design featuring scintillating light guides and an array of photodetectors underneath consist briefly in: producing a detailed square islands pattern on SU-8 over the photodetectors array; deposition of aluminum which will fill the spaces in the pattern; complete removal of the SU-8; scintillator placement inside the cavities by deposition; and an aluminum layer deposition on the top of the structure.

Fig. 2 shows a schematic representation of the detector structure. In this work, the photodetectors consist on an array of CMOS photodiodes $\left(\mathrm{n}^{+} / \mathrm{p}\right.$-substrate junctions). The scintillators are placed above the photodetectors. In this case, the chosen scintillator was thallium doped cesium iodide (CsI:Tl), due to its high light yield, relatively high density and atomic number of its elements, which is necessary in order to absorb the x-rays [6]. The thallium concentration should be around $0.03 \mathrm{~m} / \mathrm{o}$ and the evaporation rate $4 \mu \mathrm{m} / \mathrm{min}$ [7]. 


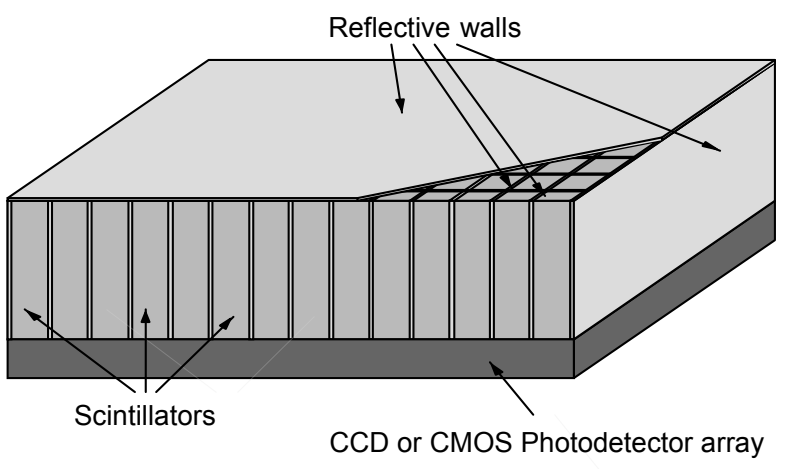

Figure 2. Schematic representation of the detector structure.

A reflective material is used to coat the scintillator. It works like a light guide avoiding visible light dispersion and interference between each neighbor pixel, thus minimizing cross-talking [6]. Moreover, it improves the spatial resolution as well as it increases the intensity of the transmitted light to the photodetectors. In addition, the amount of the incoming $\mathrm{x}$-ray radiation can be reduced while keeping the same sensitivity of the photodetectors signal readout.

The $\mathrm{x}$-rays cross the reflective material placed on the top and reach the scintillator, where they are absorbed. For each $\mathrm{x}$-ray absorbed photon, many visible light photons are produced, traveling in all directions. Some of them arrive directly at the photodetector, while others reach the reflector. After some reflections, disregarding the losses in the reflection, almost all the visible light photons reach the photodetector.

Aluminum was chosen for the reflective walls since this material has relatively low density and low atomic number, thus allowing the penetration of the x-rays [6].

\section{A. Scintillator Array}

The following paragraphs explain the scintillator array fabrication inside the aluminum walls. Sacrificial layers of MicroChem OmniCoat ${ }^{\mathrm{TM}}$ and SU-8 are spun over the CMOS photodetector array (Fig. 3(a)). SU-8 is chosen as it enables deep structures with very low sidewall roughness, which is suitable for the required cavities. Moreover, the patterning of the SU-8 implies a low cost process.

The SU-8 is exposed to UV light and after exposure, a suitable solvent (as MicroChem Developer) dissolves the unexposed resist. SU-8 columns are formed on top of the photodetectors. After this step, the OmniCoat must be also developed (Microposit MF-319 Developer may be used) (Fig. 3(b)).

The next step is to deposit, by PVD (Physical Vapor Deposition), the aluminum layer over the entire array (Fig. 3(c)).

After that, the SU-8 and the OmniCoat are removed with the MichoChem Remover PG and therefore the aluminum on top of those columns (Fig. 3(d)).

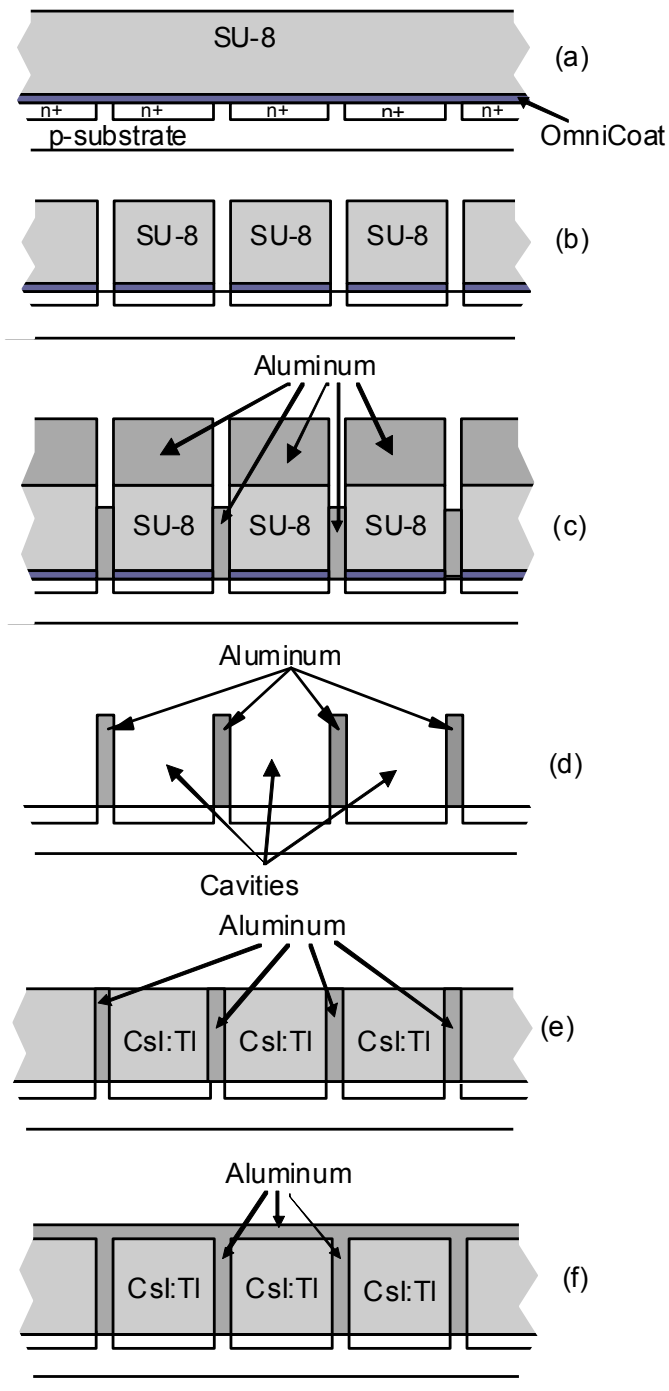

Figure 3. Fabrication steps of the scintillating light guides [6]: (a) the CMOS photodetectors array is spin-coated with OmniCoat and SU-8; (b) after exposure to UV light, a suitable solvent is used to dissolve the unexposed resist and another dissolves the OmniCoat; (c) an aluminum

layer is deposited over the entire array; (d) SU-8 and OmniCoat are removed along with the aluminum on top of it; (e) the scintillator is placed inside the cavities; and (f) a final aluminum layer is deposited.

Following, the scintillator is placed inside the cavities by PVD (Fig. 3(e)).

Finally, an aluminum layer is deposited, by PVD, on top of the scintillator (Fig. 3(f)). This step is performed after a polishing procedure in order to remove the CsI:Tl deposited on top of the aluminum walls, as well as to get an uniform surface with low surface roughness, eliminating irregularities.

\section{B. Detection System}

The main difference in the readout circuit of a visible light imager and an x-ray imager is that in the first one it is possible to read the pixels sequentially and in the second one it is not possible [8]. In a x-ray imager, all the pixels of 
the matrix must be read at the same time, synchronized with the x-ray tube that produces the x-rays. By the other hand, the image storage must be as quick as possible, once the radiation is harmful to the patient. In this device design, a circuit amplifies and holds the entire pixel matrix values at the same time, in order to be possible to later read them in a sequential way [8]. Each pixel has its own readout and processing electronics, reducing the noise and increasing the signal to noise ratio of the detector.

In this work we used a commercially available digital image sensor, but in a near future it will be replaced by our own device.

\section{DEVICE FABRICATION}

This section describes how the device, used to obtain the preliminary experimental results, was fabricated.

\section{A. SU-8 Processing}

- The MicroChem SU-8 100 was used. Using a final rotation speed of $2900 \mathrm{rpm}$, a thickness of about $100 \mu \mathrm{m}$ was obtained.

- The soft bake program followed was 10 min at $65^{\circ} \mathrm{C}$, $120 \mathrm{~min}$ at $95^{\circ} \mathrm{C}$ and cool down above hotplate for 1 hour. The samples were allowed to relax over night.

- In the exposure setup that was used, the optimum exposure was 60 seconds. The mask used in this step was cellulose acetate (regular transparency).

- $\quad$ PEB was performed at $95^{\circ} \mathrm{C}$ for 20 minutes, followed by cool down on the hotplate for 1 hour.

- The samples were developed for $10 \mathrm{~min}$ on MicroChem Developer.

\section{B. Depositions}

A $50 \mu \mathrm{m}$ thick aluminum layer was deposited (Fig. 4). Following, the SU-8 was lifted-off in Remover PG, and a layer of CsI:Tl was deposited with $50 \mu \mathrm{m}$ thickness.

\section{Experimental Setup}

- Spin coater (Laurell Technologies WS-650 LITE Series Spin Processor).

- Precision hotplate (Präzitherm PZ 28-2 EB, from Harry Gestigkeit GmbH).

- $\quad \mathrm{UV}$ exposure equipment.

- $\quad$ PVD equipment.

- Optical microscope (NIKON) with color videocamera (SONY CCD-IRIS) and computerized workstation with DVTools.

- $\quad$ X-Ray equipment (PHILIPS X'Pert, Fig. 5).

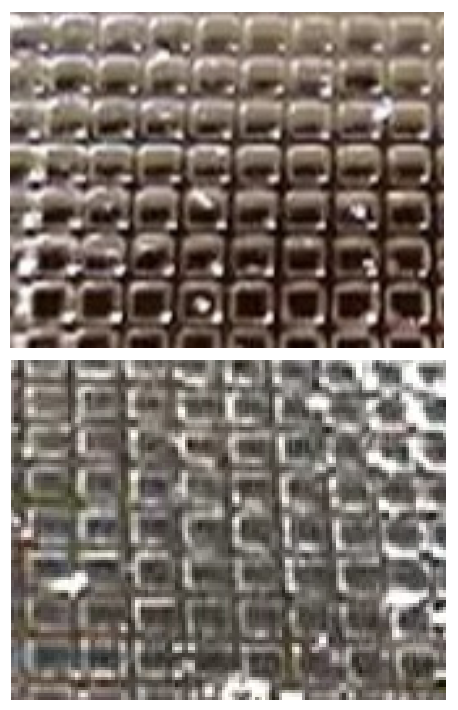

Figure 4. Images obtained after aluminum deposition (on top) and after removal of SU-8 (below).

\section{EXPERIMENTAL RESUlTS}

X-ray tests were performed using the PHILIPS X'Pert equipment (Fig. 5) The sensor with the CsI:Tl and aluminum layers was placed in the path of the x-ray beam and connected to a computer via USB port. A video was recorded. This procedure was repeated with a pin of $0.5 \mathrm{~mm}$ diameter placed close to the chip in the x-ray path (Fig. 6).

The CMOS detector array has $176 \times 144$ pixels with $35 \mu \mathrm{m} \times 35 \mu \mathrm{m}$ pixel size. In Fig. 6 it is possible to see a small amount of quantum noise. This is due to the fact that some x-rays cross the scintillator and hit directly on the CMOS detectors. It can be solved by increasing the thickness of the scintillator.

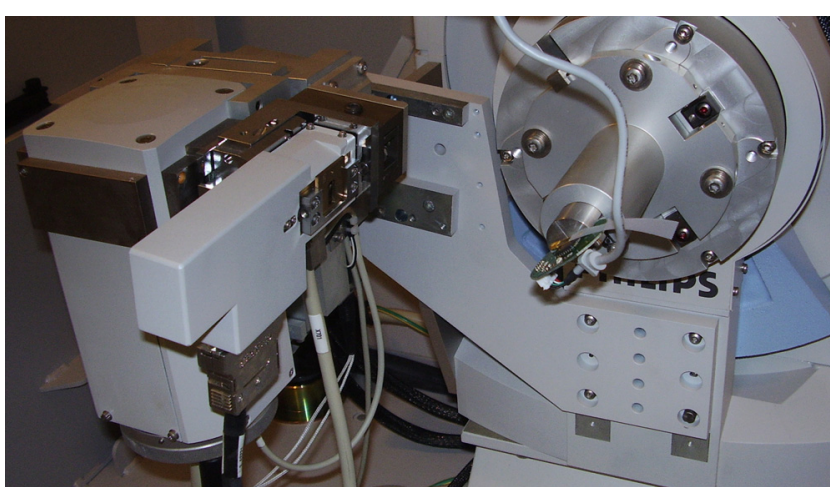

Figure 5. Experimental setup for $\mathrm{x}$-ray imaging. Detail of PHILIPS $\mathrm{X}$ 'Pert equipment with the digital imaging sensor mounted on the $\mathrm{X}$-ray beam path. 

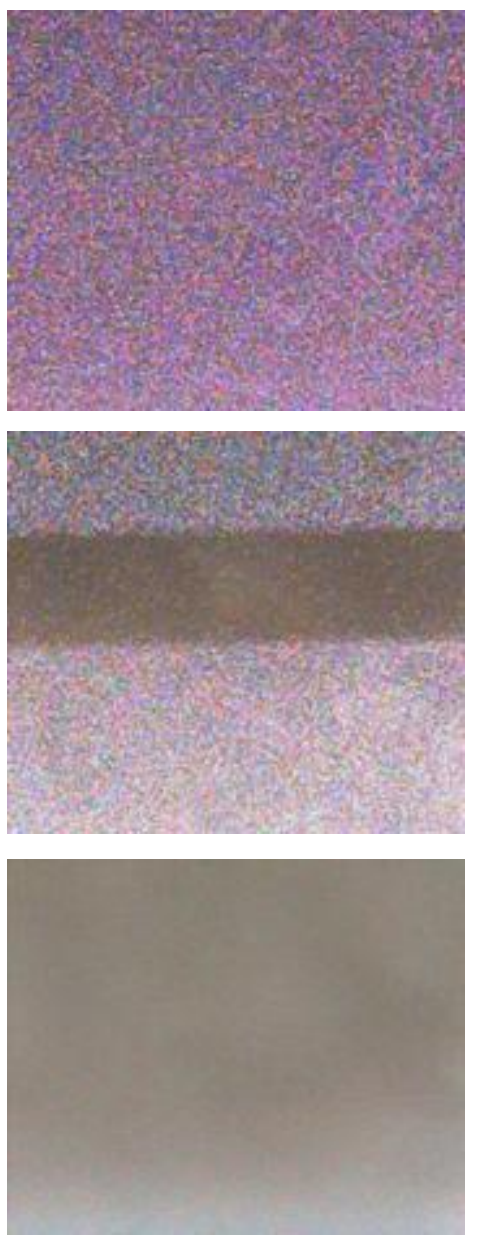

Figure 6. Images obtained without (on top) and with (middle) a pin in the $\mathrm{x}$-ray beam path, and also with the x-ray source turned off (bottom).

The main problem encountered was the complete removal of unexposed SU-8 from the narrow cavities where the aluminum is to be deposited in. The optimum developing of SU-8 revealed itself somewhat difficult. The consequences are, in the lift-off step, the release of aluminum, which might have been deposited in SU-8 residue. This fact will be improved by using OmniCoat and ultrasonic bath.

\section{CONCLUSIONS}

The spatial resolution of x-ray detectors based on scintillating crystals for digital radiography can be improved by confining the scintillator with a reflective material such as aluminum. A method for preparing cavities of reflective material, where the scintillator will be placed has the advantages of low cost and the regular shape, homogeneity and reproducibility of the cavities, was explained. Once the cavities are fabricated, the scintillator can be simply evaporated into the cavities.

The prototype obtained supports the method described and the preliminary results are promising.

The future work will comprise the use of the image sensor designed for radiology applications, briefly described in this paper, as well as the use of OmniCoat and ultrasonic bath to deal with the developing limitations of SU-8 in order to achieve higher aspect ratios thus allowing the increase in aluminum/CsI:Tl thickness.

\section{ACKNOWLEDGMENT}

The authors wish to acknowledge Mr. António de Sousa Azevedo, from Dept. of Earth Sciences, for allowing and helping us using the Phillips X'Pert equipment.

The authors also thank the funding of the portuguese ministry of Economy and Inovation - program PRIME SIUPI.

\section{REFERENCES}

[1] J. Yorkston, "Recent developments in digital radiography detectors," Nuclear Instruments and Methods in Physics Research, vol. A 580, pp. 974-985, June 2007.

[2] J. G. Rocha and S. Lanceros-Mendez, "X-ray imaging matrix with light guides and intelligent pixel sensors, radiation or high energy particle detector devices that contain it, its fabrication process and its use," patent WO2007046010.

[3] P. Kleimann, J. Linnros, C. Frojdh, and C. S. Petersson, “An x-ray imaging pixel detector based on a scintillating guides screen," IEEE Tran. Nucl. Sci., vol. 47, pp. 1483-1486, August 2000.

[4] C. P. Allier, R. W. Hollander, et al., "Thin photodiodes for a neutron scintillator silicon-well detector," IEEE Tran.Nucl. Sci., vol. 48, pp. 1154-1157, August 2001.

[5] J. G. Rocha, C. G. J. Schabmueller, et al., "X-ray detector based on a bulk micromachined photodiode combined with a scintillating crystal," Journal of Micromechanics and Microengineering, vol. 13, pp. S45-S50, July 2003.

[6] J. G. Rocha, G. Minas, L. M. Goncalves and S. Lanceros-Mendez, "Scintillating microcavities for X-ray imaging sensors," MicroMechanics Europe Workshop, pp. 149-152, September 2006.

[7] I. Fujieda, G. Cho, et. al., "X-ray and charged particle detection with CsI(Tl) layer coupled to a a-Si:H photodiode layers," IEEE Trans. Nucl. Sci., vol. 38, pp. 255-262, April 1991.

[8] J. Silva, S. Lanceros-Mendez, G. Minas, and J. G. Rocha, "CMOS xray image sensor array," Proceedings of ICECS, pp. 1067-1070, December 2007. 